\title{
Targeting the Folate Receptor: Effects of Conjugating Folic Acid to DOX Loaded Polymeric Micelles
}

\author{
Mohamed A. Elkhodiry ${ }^{\mathrm{a}}$, Ghaleb A. Husseini ${ }^{\mathrm{a},{ }^{*}}$ and Diana Velluto ${ }^{\mathrm{b}}$
}

${ }^{a}$ Department of Chemical Engineering, College of Engineering, American University of Sharjah, Sharjah, United

Arab of Emirates, ${ }^{b}$ Diabetes Research Institute, Miami, FL, USA

\begin{abstract}
In this paper, we report on a potential cancer drug delivery system that utilizes the ligand targeting of the folate receptor. Our drug delivery system consists of Pluronic-P105 micelles, targeted with folic acid moieties. A melanoma folate positive (FR+) (B16-F10), and a fibroblast folate negative (FR-) (NIH-3T3) cell lines are used to compare the cellular accumulation of a chemotherapeutic drug (Doxorubicin) when the delivery is mediated by folated Pluronic P105 micelles. In order to obtain a proper comparison, we corrected for the quenching of Doxorubicin by folic acid molecules and illustrated the significant effect of quenching on the analysis of similar systems. Results show an $80 \%$ increase in the accumulation of the antineoplastic agent in the FR+ cell line, when compared to the FR- cell line, thus providing evidence that the efficacy of Pluronic micelles, as drug delivery vehicles, can be enhanced via folic acid targeting.
\end{abstract}

Keywords: Doxorubicin, micelles, folate, active targeting, quenching, B16-F10, melanoma.

\section{INTRODUCTION}

In the 1950s, a soil bacterium strain, Streptomyces Peucetius, was used as a source of a novel cancer treating anthracycline compound called Daunorubicin [1-3]. Daunorubicin which showed unprecedented efficacy in killing cancer cells, was later identified as a primary cause of cardiac toxicity $[2,4]$. In an attempt to improve over such drawbacks, a similar bacterial strand was genetically modified to produce Doxorubicin (DOX), another anthracycline compound with a higher therapeutic index and is one of today's most widely administered chemotherapeutic agents [2, 5-7].

The exact mechanism by which the drug functions remains a debated topic. DOX's unique anticancer activity was attributed to its heteroaromatic planar structure's ability to stabilize Topoisomerase II [2]. Increasing the stability of Topoisomerase II prevents the recoiling of cancer cell DNA after replication which initiates a DNA damage response and hence, stops tumor growth [6]. However, other studies suggest that DOX is, in fact, a Topoisomerase II inhibitor that occupies the areas between base pairs of DNA and decreases the DNA flexibility, thus increasing the torsional force and resulting in structural damage to the DNA of the cancer cells $[1,6,7]$. Despite continuing concerns over the drug's cardiotoxicity, DOX has been widely used in treating various types of tumors including leukemia, lymphoma, breast and lung cancers $[2,8]$.

In the late 1980s, advancements in nanotechnology along with the need to eliminate DOX's cardiotoxic side effects inspired the utilization of polymeric nanocarriers in the delivery of this and other agents [9]. Amphiphilic block copolymers with a certain concentration dissolved in a solvent, which physically accumulate in one of the blocks, lead to the formation of polymeric micelles that encapsulate the drug [9-11]. The block copolymers aggregate to satisfy their chemical affinities, making it one of the easiest nanocarriers to synthesize. Today, after years of research, polymeric

micelles are among the most commonly researched drug nanocarriers [12]. This can be mainly attributed to their spontaneous selfassembly, suitable size and biocompatibility $[11,13]$.

Polymeric micelles range between 10 and $100 \mathrm{~nm}$ in size, making their structure suitable to take advantage of the enhanced permeability and retention (EPR) effect [14-16]. Nano-vehicles used in cancer treatment are capable of accumulating preferentially

"The published manuscript is available at EurekaSelect via http://www.eurekaselect.com/openurl/ content.php?genre=article\&doi=[https://doi.org/10.2174/1871520616666160219161600].” 
in the diseased area due to the defective vascular architecture present in most solid tumors [17]. This enables these stealth micelles to be "passively" entangled inside the leaky capillaries of the tumor, allowing for the slow release of their therapeutic contents. Thus, the EPR effect allows the micelles and their encapsulated therapeutic chemicals to remain and accumulate in tumor tissues for an extended period of time, possibly reducing their cytotoxicity in other parts of the body. This allows the creation of a drug delivery system that targets malignant tumors leaving healthy tissue with low drug concentration and hence less unwanted side effects [3]. This approach is known as passive targeting. It is important to note that while the EPR effect is dependent on the size of liposomes, it is also affected by the particle composition, and the tumor environment tissue characteristics including permeability, tissue abnormality, lymph drainage, etc.

The conjugation of a targeting moiety on the surface of the micelles, in order to increase the nanoparticle's affinity towards malignant tissue, has been studied for more than a decade $[18,19]$. This modification of the micellar surface can be utilized to enhance the efficiency of the treatment. The type of the moiety to be conjugated depends on the type of receptor overexpressed on the surface of the tumor cells under investigation [20]. This type of surface modification is termed active or ligand-targeting and is defined as the use of targeting ligands for enhanced delivery and accumulation of nanoparticles to the diseased site [16].

Choosing the proper moiety for targeting is essential because of its effect on cellular uptake, affinity, circulation time, and extent of extravasation into the tumor [21]. Ligands used as targeting moieties include: antibodies, peptides, aptamers, hormones, and low molecular weight compounds $[3,16]$. The most commonly used low molecular weight compound is folic acid which is a naturally occurring vitamin overexpressed in a variety of malignancies including breast, ovarian, and skin cancers [20]. Its use in ligand targeting is attractive because of its small size and the simple conjugation schemes it requires [3]. In an attempt to increase the efficacy of ovarian cancer targeting, Werner et al. [22] created a folate modified nanoparticle that showed higher therapeutic effect on the metastasis in mice. Similarly, Zhu et al. [23] created folated micelles that were able to specifically target hepatic carcinoma cells and achieve a significantly higher drug concentration in implanted tumor tissue compared to their non-targeted counterparts.

In this paper, we will examine the effect of conjugating folic acid to Pluronic P105 micelles on two cell lines: a folate positive (FR+) B16-F10 and a folate negative (FR-) NIH-3T3 cell lines.

\section{MATERIALS AND METHODS}

\section{Micelles Preparation}

P105-FA was synthesized using 1,1-carbonyldiimidazole (CDI, Sigma Aldrich). Approximately $1.026 \mathrm{~g}$ of folic acid (FA, Sigma Aldrich) was dissolved in $100 \mathrm{ml}$ of dried DMSO [26]. Then, $0.414 \mathrm{~g}$ CDI was added and allowed to react for 4 hours under dark conditions at room temperature. After the activation of the FA, $30.2 \mathrm{~g}$ of Pluronic P105 (BASF), which was dried overnight under vacuum, was added to the above solution. The activated FA and Pluronic P105 were allowed to react for 20 hours in darkness at room temperature. At the conclusion of the reaction, the product was dialyzed (Spectra Millipore MWCO 3500) against DMSO for 2 days and then against DD-water for 2 additional days. The purified product was then lyophilized and stored at $-20^{\circ} \mathrm{C}$. The formation of P105-FA was confirmed using 1HNMR. The 1HNMR spectra were recorded on a Bruker 400 UltraShield spectrometer at room temperature in $\mathrm{CDCl}$. For cell experiments, $\mathrm{P} 105-\mathrm{FA}$ was dissolved in PBS, $\mathrm{pH} 7.4$, to make a final concentration of 5 wt. \% (5 wt. \% P105-FA, 95 wt. \% phosphate buffered saline (PBS)). Dox (Sigma Aldrich) was introduced into the micelles by mixing at room temperature to a final concentration of $10 \mu \mathrm{g} / \mathrm{ml}$. The drugloaded micellar solution was degassed overnight before being used in cell experiments.

The mean diameter and polydispersity of the self-assembled micellar structures in aqueous media were defined by dynamic light scattering (DLS, Zetasizer Nano ZS, Malvern Instrument Ltd., U.K.), with the concentration of the polymers at $5 \%$. 
Cryotransmission electron microscopy (cryo-TEM) was also used to investigate the morphology of the aggregates in water. The specimens for analysis were prepared by the application of a drop of the aqueous aggregate suspension on a micro-copper grid coated with a porous carbon film. Excess suspension was blotted away, resulting in a 30-100 nm thick film that spans the holes of porous carbon support. The sample was then rapidly vitrified by immersion in liquid ethane, transferred to the cryo-electron microscope (Philips CM12 FEI, Eindhoven, Netherlands) operating at $80 \mathrm{kV}$ in transmission mode at a temperature not allowed to exceed -160 ${ }^{\circ} \mathrm{C}$. Images were recorded on a CCD camera with minimum electron dose at a nominal magnification of up to $3500 \mathrm{x}$.

\section{Cell Culture}

B16-F10 melanoma cells, derived from C57BL/6 mice, and NIH-NIH-3T3 cells (standard fibroblast cell line), were cultured at 37 ${ }^{\circ} \mathrm{C}$ in a humidified 5\% $\mathrm{CO}_{2}$ incubator in Dulbecco's modified Eagle's medium (DMEM, from Invitrogen, Basel, Switzerland) supplemented with $10 \%$ fetal bovine serum (Invitrogen) with penicillin at $100 \mathrm{U} / \mathrm{ml}$ and streptomycin at $100 \mathrm{mg} / \mathrm{ml}$ (both from Invitrogen).

\section{Cell Treatment}

Briefly, $5 \times 10^{4}$ of each cell line was plated into a 24-well plate containing $500 \mathrm{ml}$ of DMEM and $10 \%$ fetal bovine serum. After 24 $\mathrm{h}$, when the cells were $70-80 \%$ confluent, DOX loaded folated micelles and non-folated micelles were added to the media and incubated with both B16-F10 and NIH-NIH-3T3 cells for 15 and $30 \mathrm{~min}$ at $37^{\circ} \mathrm{C}$. The incubation media was then removed and cells were extensively washed with PBS. Cells were trypsinised, washed and suspended in PBS for immediate flow cytometric analysis.

To compare between the DOX concentrations in our samples, the linear proportional relation between fluorescence intensity and concentration expected by Beer's law was used. The difference between the sample intensity and the background intensity was calculated for each case and the ratios between such differences provided an accurate measure of the ratios of DOX between the different samples. P-values were calculated using a student ttest.

\section{RESULTS AND DISCUSSION}

We have reported earlier that ultrasound can be used to release DOX from non-targeted [24, 25] and targeted micelles [26, 27]. In vivo experiments showed a slight increase of Dox accumulation when comparing the sonicated and control tumors (an increase of approximately 20\%) [28-30]. In order to improve this drug delivery system, we opted to conjugate a folate moiety to the surface of Pluronic P105 micelles. The idea is to use all three targeting techniques to our advantage (namely passive, ligand and triggered targeting) in future in vivo experiments.

In characterizing the P105-FA, NMR spectra showed a broad peak at $3.7 \mathrm{ppm}$ (attributed to the PEG backbone) and characteristic peaks attributed to folic acid at 2.3,6.6, 7.6 and $8.6 \mathrm{ppm}$. These NMR peaks were used to calculate a yield of $48 \%$. To characterize the micelles, we measured the average hydrodynamic diameter by dynamic light scattering measurements (DLS) at a fixed angle of $90^{\circ}$. The average diameter of these micelles was measured to be $10.2+2.2 \mathrm{~nm}$. This size was also confirmed by cryo-TEM. The poly dispersity of our samples was $24.9 \% \pm 8.4 \%$. Having the micelles in this size range ensures that the designed drug delivery system can take advantage of the EPR effect; the micelles are small enough to escape the blood stream through the tumor highly permeable vasculature and accumulate in the tumor tissue. This has been previously studied and it has been shown that a nanocarrier size range of $10-200 \mathrm{~nm}$ is the optimum range for passive targeting [16, 31, 32]. 


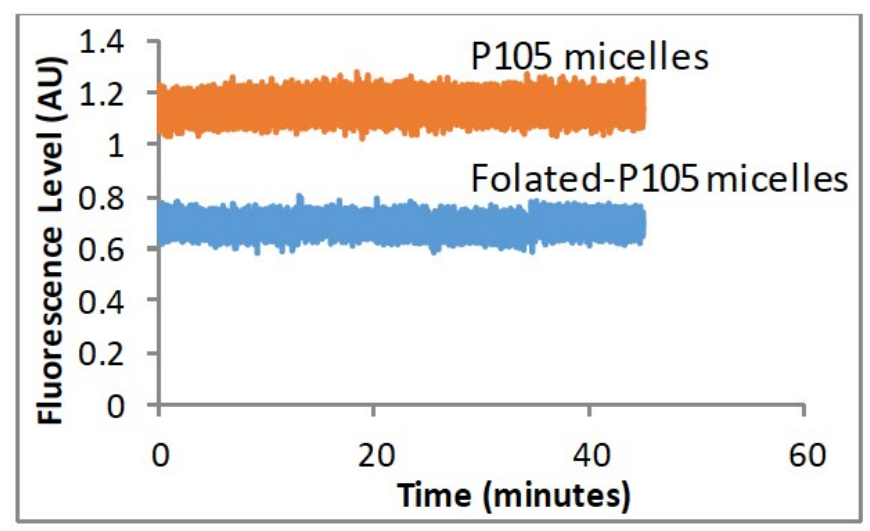

Fig. (1). Fluorescence of Dox in P105 non-targeted and folate-targeted micellar solutions as a function of time. The figure shows the stability of both types of nanocarriers.

Additionally, we have measured the stability of both folated and non-folated liposomes in media for 45 minutes. Dox exhibits a decrease in fluorescence when transferred from the poly-propylene hydrophobic core of the micelles to the aqueous environment surrounding the micelles [33]. This decrease in fluorescence upon changing the non-polar to polar micro-environments of the agent has been extensively studied and documented, and has been utilized to study the ultrasonic release of Doxorubicin from folatetargeted P105 and non-folated micelles [34,35] and to model the kinetics of acoustically activated drug delivery [36-41]. Fig. 1 shows the fluorescence of Dox encapsulated in folated and non-folated micelles as a function of time. The figure shows that the fluorescence level is constant for 45 minutes, hence providing evidence of the micellar stability in growth media for the duration of our in vitro experiments.In order to evaluate the effect of the presence of a folate receptor on the cellular uptake of the folated micelles, flow cytometric analysis was performed to measure the cellular uptake of DOX by two cell lines: NIH-3T3 fibroblast, which is folate negative (FR-), and B16-F10 melanoma, which is folate positive (FR+). Flow cytometric analysis performed after 15 minutes of incubation was used to evaluate the efficacy of DOX loaded folated micelles as an active targeting therapeutic system. Fig. 2 (left) shows that the B16-F10 cellular drug uptake is significantly higher compared to the NIH-3T3 cells ( -value $=0.000112)$; the presence of the folate receptor on the surface of the B16-F10 cells and the activation of its pathway caused a $70 \%$ increase in cellular uptake when compared to the uptake of NIH-3T3 cells.
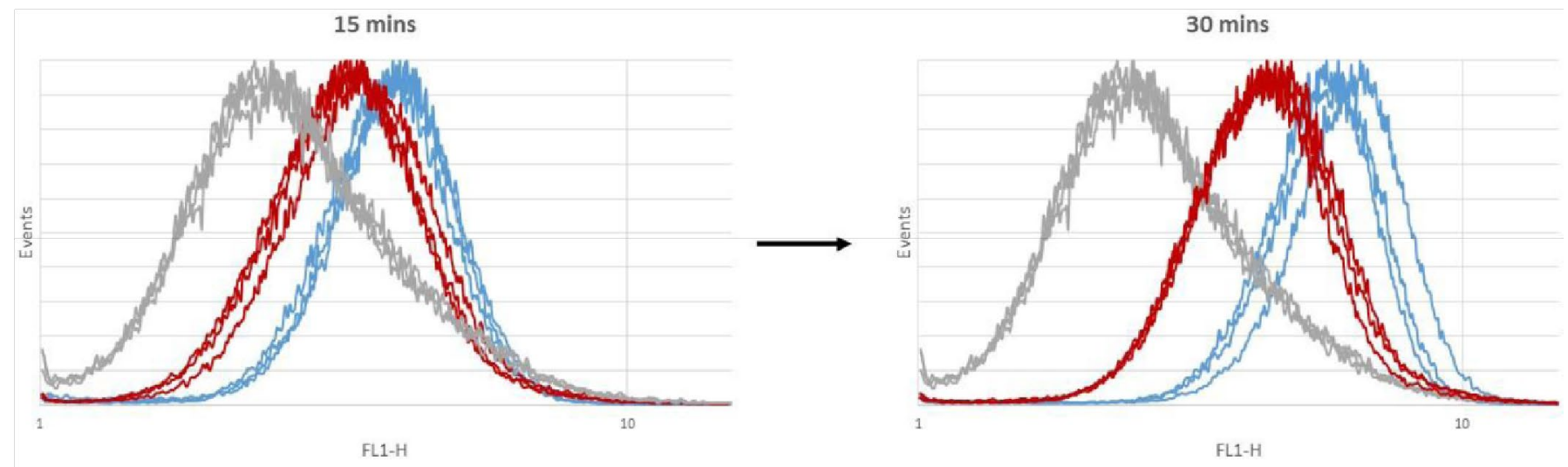

Fig. (2). Flow cytometric analysis results, for NIH-3T3 (red) and B16-F10 (blue) cells, 15 and 30 minutes after incubation with folated micelle. The figure shows three replicated for each condition at both time points. The average peak fluorescence for the NIH-3T3 and B16-F10 cell lines incubated for 15 minutes with Dox encapsulated in folated-micelles are $3.6 \pm 0.15$ and $4.1 \pm 0.11$, respectively. The average accumulation increases after 30 minutes of incubation inside these targeted micelles to reach $4.43 \pm 0.092$ (for the FR- cell line-NIH-3T3), and $6.00 \pm 0.435$ for the (FR+ cell line-B16-F10). 
Our results complement Varshosaz et al. [41] results using folate activated polymeric micelles and Kang et al.'s [42] results using folate activated liposomes to treat B16-F10 cells which confirms the ability of the folate ligand to differentially target the melanoma cells. The results also match previous micellar studies on Bel-7402 hepatoma cells [23], KB cells [43], MATB III breast cancer cells, and C6 glioma cells [44] emphasizing the importance of folate as a common tumor targeting moiety overexpressed on the surface of several cancer cell lines.

Increasing the incubation time from 15 to 30 minutes increases the cellular uptake of the folated micelles in both cell lines as shown in Fig. 2 (right). The role of the folate receptors exhibits a slight increase; the B16-F10 cells that show 1.7 times increase in the concentration of DOX when compared to NIH-3T3 cells after 15 minutes of incubation, show 1.8 times the concentration of DOX recorded in NIH-3T3 cells after 30 minutes of incubation. This further confirms the role of the folate receptor in facilitating drug uptake via receptor-mediated endocytosis but also reflects the process' dependence on time and the importance of choosing an optimum incubation time in future studies. It is expected that increasing the incubation time beyond 2 hours will result in a lower drug concentration in tumor cells as shown by Amin et al. [45] and, therefore, it is essential to optimize the incubation to achieve the maximum efficiency of this drug delivery system.

The previously presented data accentuate the folic acid ability as a ligand to target the folate receptor on surfaces of tumor cells, yet upon comparing the cellular uptake of the synthesized folated micelles to non-folated micelles the results are seemingly contradicting. To demonstrate the effective ligand interaction, our drug delivery system has to be compared to a system that lacks either the receptor or the ligand. Exposing the cell cultures to non-targeted micelles (micelles without FA moiety) provides a control sample that lacks the ligand and therefore, should not exhibit any active targeting character. As shown in Fig. 3, the fluorescence intensity of the B16F10 cells treated with non-folated micelles are 9 times more than that of the same cells treated with the same concentration of folated micelles. This opposes both theoretical expectations and previous work reported in literature $[23,43,46]$. Theoretically, a folated nanocarrier causes a higher cellular uptake when compared to a non-targeted similar nanocarrier (when incubated in the same folate receptor-positive cell line) due to the instigation of receptor mediated endocytosis processes, as long as receptor saturation has not been reached [16, 47-49]. This has been proven in vitro in different folate $(+)$ cell lines including KB [50, 51], M109 lung carcinoma [19], HeLa cervical carcinoma [48], Bel 7402 hepatic carcinoma [52], and B16-F10 cells [41]. The results shown in Fig. 3 clearly indicate the existence of an entirely different trend.

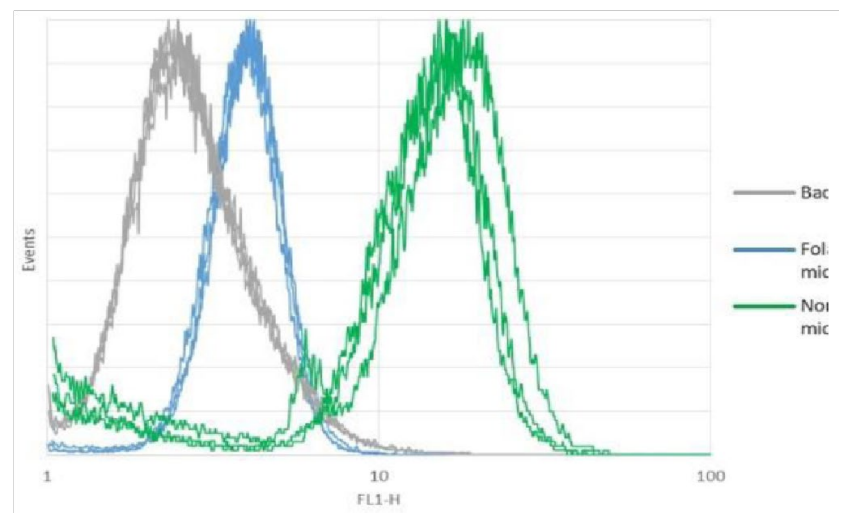

Fig. (3). Flow cytometric analysis results of folated vs. non-folated micelles for Dox accumulation in the B16-F10 cell line. The figure shows three replicated per condition. The average peaks of Dox accumulation after 30 minutes of incubation with non -targeted and targeted micelles are $17.78 \pm 1.43$, and $4.1 \pm 0.11$, respectively. 
Before correction

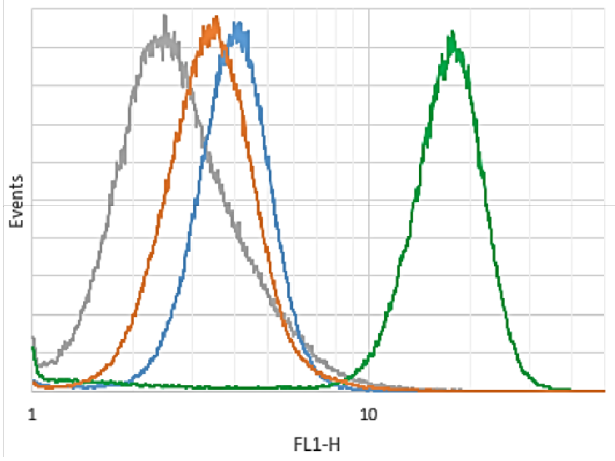

After correction

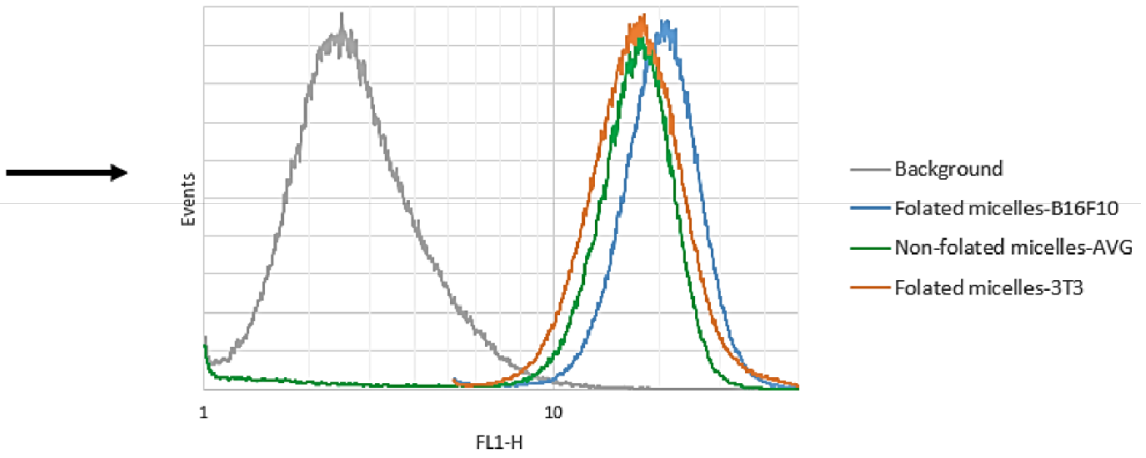

Fig. (4). Flow cytometric analysis results before and after correcting for quenching. The correction was made by multiplying the average fluorescence of the three folated micelles replicates by 5.11 .

This unexpected behavior can be attributed to a chemical interaction between DOX and folic acid causing a quenching effect on the fluorescence of DOX. This has been studied by Santra et al. [53] who created a FOL-DOX conjugate in an attempt to increase the efficacy of DOX delivery. The resultant conjugate had a fluorescence intensity 5 times lower than DOX. Husseini et al. [54, 55] went on to the study the existence and nature of the quenching phenomenon between folic acid and DOX molecules in different $\mathrm{pH}$ media, including at physiological $\mathrm{pH}$. A similar quenching effect was observed at the different $\mathrm{pH}$ conditions. Moreover, fluorescence emission showed a decrease in the folic acid peak at $395 \mathrm{~nm}$ and the DOX peak at $520 \mathrm{~nm}$ and an emergence of a new peak at $430 \mathrm{~nm}$, suggesting the presence of FOL-DOX conjugates. Their study used free folic acid in PBS (not folated micelles as is the case in this study). The presence of the P105 molecules/micelles attenuates the interaction between the two molecules and as a result, the fluorescence correction factors previously reported by Husseini et al. are not accurate for this study. However, this quenching phenomenon should be accounted for to properly compare our results.

One way to account for the DOX/folic acid quenching effect is to assume that the concentration of the drug in the B16-F10 cells with non-folated micelles is the same as that of the NIH-3T3 cells with folated micelles. In both treatment groups, receptor mediated endocytosis is absent and the micelles enter the cells by other mechanisms. The drug concentration in B16-F10 (average peak fluorescence values $=17.78 \pm 1.43)$ and NIH-3T3 (average peak fluorescence values $=16.62 \pm 1.12)$ incubated with nonfolated micelles and was found to be statistically insignificant ( $\mathrm{p}$-value $=0.8976$ ), showing similar accumulation levels of the drug in the cells using non-targeted P105 micelles as drug delivery vehicles.

Therefore, if the fluorescence intensity of the NIH-3T3 cells with folated micelles is simply multiplied by a factor to make it equal to that of the B16-F10 cells with non-folated micelles, then this factor would be the correction factor needed to account for quenching under the same folic acid, DOX, and P105 concentrations of any other sample. This strategy gives a correction factor of 5.11 causing the histogram shift shown in Fig. 4 and resulting in a cytometric profile similar to that of the B16-F10 liposomal system with and without folate reported by Kang et al. [41]. In Kang and coworkers' drug delivery system, the fluorescent agent, encapsulated in the nanocarriers, is a dye that does not interact with folic acid molecules, hence providing an accurate measure of the efficacy of using folate ligands against B16-F10 cells. The 5.11 correction factor we calculated matches the 5-fold quenching of DOX fluorescence observed by Santra et al. [53], again confirming the formation of DOX-FOL conjugates. However, the exact nature of the association between the folic acid and the DOX remains unclear. 
The implications of these observations can be extended to the folate competitive inhibition studies. In order to further confirm the role of the folate receptor in the active targeting process, many studies add a known amount of free folic acid to the system to competitively inhibit the ligand-receptor facilitated uptake process. The free folic acid (usually present in high concentrations) binds to the folate receptor reducing the likelihood of the folated micelle binding to a free folate receptor. As a result, in such highconcentration-folic-acid media, the concentration of the drug in FR+ cells after incubation will be lower compared the folic acid free medium [56, 57]. However, this reduction in uptake, observed in the reduced fluorescence intensity, is not necessarily due to competitive inhibition alone. We believe that if DOX is the chemotherapeutic agent of choice, and in the presence of a high concentration of free folic acid, its fluorescence will be lower due to quenching [54, 55]. The higher the concentration of free folic acid in the treatment media, the higher is the validity of the hypothesis that the formation of the FOL-DOX conjugates provide an explanation for the decrease in fluorescence intensity of the FR+ samples upon adding folic acid as a competitive antagonist as reported previously [43].

However, the reason why this behavior has never been observed by other researchers studying the same system remains unclear. This may require further molecular analysis of the FOL-DOX conjugates that could have been enhanced by the particles' concentrations, the FA-P105 synthesis protocol, or the flow cytometry conditions. Further analysis of this quenching effect in the presence of different polymers is also required in hope of providing proper correction factors in cytotoxicity analysis of folated nanocarriers.

The results reported herein show the utility of using folatetargeting to enhance the efficacy of acoustically-activated Dox delivery from P105 micelles. We have reported earlier the results of using non-targeted P105 micelles in vitro and in vivo. Our results showed that US and micelles/Dox in a rat model showed selective reduction of tumor growth rates, but only by $20 \%$. We believed that the targeted micelles in this work have the capability of improving the efficiency/drug accumulation/tumor regression rates of the P105 (non-targeted) micelles studied earlier.

\section{CONCLUSION}

In conclusion, we reiterate the advantages of using polymer micelles as drug delivery vehicles: their attractive size, simple assembly, long shelf-live and low toxicity. More specifically, here we report on enhancing their targetability towards certain cancer cell lines via the conjugation of a targeting moiety to their surface. Folic acid is arguably the most widely researched targeting moiety because of its small size and simple conjugation technique and hence was used in this work. Furthermore, our data highlights the need to correct for the quenching of Dox by folic acid molecules. Our results show that drug delivery systems can enhance their efficacy by utilizing receptor-mediated endocytosis. More research is currently being undertaken to see the effect of adding ultrasound to these folate-targeted micelles on both cells lines. The ultimate goal is to reach an optimal multimodal drug delivery system that helps mitigate the side effects of conventional chemotherapy.

\section{CONFLICT OF INTEREST}

The author(s) confirm that this article content has no conflict of interest.

\section{ACKNOWLEDGEMENTS}

The authors would like to acknowledge the funding from the American University of Sharjah Faculty Research Grant 1 (AUS/FRG1-2012), Patient's Friends Committee-Sharjah, and Al Qasimi Foundation.

\section{REFERENCES}

[1] Yang, F.; Teves, S. S.; Kemp, C. J.; Henikoff, S. Doxorubicin, DNA torsion, and chromatin dynamics. BBA-Rev. Cancer, 2014, $1845(1)$, 84-89.

[2] Rivankar, S. An overview of doxorubicin formulations in cancer therapy. J. Cancer Res. Ther., 2014, 10(4), 853-858. 
[3] Bertrand, N.; Wu, J.; Xu, X. Y.; Kamaly, N.; Farokhzad, O. C. Cancer nanotechnology: The impact of passive and active targeting in the era of modern cancer biology. Adv. Drug Deliver. Rev., 2014, 66, 2-25.

[4] Shi, Y.; Moon, M.; Dawood, S.; McManus, B.; Liu, P. P. Mechanisms and management of doxorubicin cardiotoxicity. Herz, 2011, 36(4), 296-305.

[5] Mizutani, H.; Tada-Oikawa, S.; Hiraku, Y.; Kojima, M.; Kawanishi, S. Mechanism of apoptosis induced by doxorubicin through the generation of hydrogen peroxide. Life Sci., 2005, 76 (13), 1439-1453.

[6] Pang, B. X.; Qiao, X. H.; Janssen, L.; Velds, A.; Groothuis, T.; Kerkhoven, R.; Nieuwland, M.; Ovaa, H.; Rottenberg, S.; van Tellingen, O.; Janssen, J.; Huijgens, P.; Zwart, W.; Neefjes, J. Drug-induced histone eviction from open chromatin contributes to the chemotherapeutic effects of doxorubicin. Nat. Commun., 2013, 4, 1908.

[7] Filyak, Y.; Filyak, O.; Souchelnytskyi, S.; Stoika, R. Doxorubicin inhibits TGF- $\beta$ signaling in human lung carcinoma A549 cells. Eur. J. Pharmacol., 2008, 590 (1), 67-73.

[8] Chen, N. T.; Wu, C. Y.; Chung, C. Y.; Hwu, Y.; Cheng, S. H.; Mou, C. Y.; Lo, L.W. Probing the dynamics of doxorubicin-DNA intercalation during the initial activation of apoptosis by fluorescence lifetime imaging microscopy (FLIM). Plos One, 2012, 7(9), e44947.

[9] Miyata, K.; Christie, R. J.; Kataoka, K., Polymeric micelles for nano-scale drug delivery. React. Function. Poly., 2011, 71 (3), 227234.

[10] Gast, A.P. Polymeric micelles. Curr. Opin. Colloid Interface Sci., 1997, 2(3), 258-263.

[11] Owen, S.C.; Chan, D.P.Y.; Shoichet, M.S. Polymeric micelle stability. Nano. Today, 2012, 7(1), 53-65.

[12] Gong, J.; Chen, M.W.; Zheng, Y.; Wang, S. P.; Wang, Y.T. Polymeric micelles drug delivery system in oncology. J. Control. Release, 2012, 159(3), 312-323.

[13] Croy, S.R.; Kwon, G.S. Polymeric Micelles for Drug Delivery. Curr. Pharm. Des., 2006, 12 (36), 4669-84.

[14] Mahmud, A.; Xiong, X.B.; Aliabadi, H.M.; Lavasanifar, A. Polymeric micelles for drug targeting. J. Drug Target., 2007, 15 (9), 553-584

[15] Kataoka, K.; Harada, A.; Nagasaki, Y. Block copolymer micelles for drug delivery: Design, characterization and biological significance. Adv. Drug Deliver. Rev., 2012, 64, 37-48.

[16] Elkhodiry, M.A.; Momah, C.C.; Suwaidi, S. R.; Gadalla, D.; Martins, A.M.; Vitor, R.F.; Husseini, G.A. Synergistic nanomedicine: passive, active and ultrasound-triggered drug delivery in cancer treatment. J. Nanosci. Nanotechnol., 2015, 15, 119.

[17] Liu, T.J.; Liu, S.; Hu, X.L.; Sheng, S.H.; Huang, Y.B.; Jing, X.B. EPR effect of amphiphilic copolymer micelles observed by fluorescent imaging. Chem. Res. Chin. Univ., 2011, 27(4), 628-634.

[18] Torchilin, V.P. Targeted polymeric micelles for delivery of poorly soluble drugs. Cell. Mol. Life Sci., 2004, 61(19), $2549-2559$.

[19] Gabizon, A.; Horowitz, A.T.; Goren, D.; Tzemach, D.; Mandelbaum-Shavit, F.; Qazen, M. M.; Zalipsky, S. Targeting folate receptor with folate linked to extremities of poly(ethylene glycol)-grafted liposomes: In vitro studies. Bioconjugate Chem., 1999, 10(2), 289-298.

[20] Byrne, J.D.; Betancourt, T.; Brannon-Peppas, L. Active targeting schemes for nanoparticle systems in cancer therapeutics. Adv. Drug Deliver. Rev., 2008, 60 (15), 1615-1626.

[21] Yu, B.; Tai, H.C.; Xue, W.; Lee, L.J.; Lee, R. J. Receptor-targeted nanocarriers for therapeutic delivery to cancer. Molec. Membrane Biol., 2010, 27 (7), 286-298.

[22] Werner, M.E.; Karve, S.; Sukumar, R.; Cummings, N.D.; Copp, J.A.; Chen, R.C.; Zhang, T.; Wang, A.Z. Folate-targeted nanoparticle delivery of chemo- and radiotherapeutics for the treatment of ovarian cancer peritoneal metastasis. Biomaterials, 2011, 32(33), 8548-8554.

[23] Zhu, H.; Liu, F.; Guo, J.; Xue, J.; Qian, Z.; Gu, Y. Folate-modified chitosan micelles with enhanced tumor targeting evaluated by near infrared imaging system. Carbohydr. Polym., 2011, 86(3), 11181129.

[24] Husseini, G.A.; El-Fayoumi, R.I.; O'Neill, K. L.; Rapoport, N.Y.; Pitt, W.G. DNA damage induced by micellar-delivered doxorubicin and ultrasound: comet assay study. Cancer Lett., 2000, 154(2), 211-216.

[25] Husseini, G.A.; Myrup, G.D.; Pitt, W.G.; Christensen, D.A.; Rapoport, N.Y. Factors affecting acoustically triggered release of drugs from polymeric micelles. J. Control. Release, 2000, 69(1), 43-52.

[26] Husseini, G.A.; Velluto, D.; Kherbeck, L.; Pitt, W.G.; Hubbell, J.A.; Christensen, D.A., Investigating the acoustic release of doxorubicin from targeted micelles. Colloids Surf. B, 2013, 101, 153-155.

[27] Husseini, G.A.; Kherbeck, L.; Pitt, W.G.; Hubbell, J.A.; Christensen, D.A.; Velluto, D. Kinetics of Ultrasonic Drug Delivery from Targeted Micelles. J. Nanosci. Nanotechnol., 2015, 15 (3), 2099-2104.

[28] Staples, B.J.; Pitt, W.G.; Roeder, B.L.; Husseini, G.A.; Rajeev, D.; Schaalje, G.B., Distribution of doxorubicin in rats undergoing ultrasonic drug delivery. J. Pharm. Sci., 2010, 99 (7), 3122-3131.

[29] Staples, B.J.; Roeder, B.L.; Husseini, G.A.; Badamjav, O.; Schaalje, G.B.; Pitt, W.G. Role of frequency and mechanical index in ultrasonic-enhanced chemotherapy in rats. Cancer Chemother. Pharmacol., 2009, 64(3), 593-600. 
[30] Nelson, J.L.; Roeder, B.L.; Carmen, J.C.; Roloff, F.; Pitt, W.G. Ultrasonically activated chemotherapeutic drug delivery in a rat model. Cancer Res., 2002, 62(24), 7280-7283.

[31] Sano, K.; Nakajima, T.; Choyke, P. L.; Kobayashi, H. Markedly enhanced permeability and retention effects induced by photoimmunotherapy of tumors. ACS Nano., 2013, 7(1), 717-724.

[32] Kobayashi, H.; Watanabe, R.; Choyke, P. L., Improving conventional enhanced permeability and retention (epr) effects; what is the appropriate target? Theranostics, 2014, 4(1), 81-89.

[33] Husseini, G.A., Christensen, D.A., Rapoport, N.Y., and Pitt, W.G., Ultrasonic release of doxorubicin from Pluronic P105 micelles stabilized with an interpenetrating network of $N, N$ diethylacrylamide, $J$. Control. Release, 2002, 83(2), 303-305.

[34] Husseini, G.A.; Diaz de la Rosa, M.A.; Gebuji, T.; Zeng, Y.; Christensen, D.A.; Pitt, W.G. Release of Doxorubicin from Unstabilized and Stabilized Micelles Under the action of Ultrasound, J. Nanosci. Nanotechnol., 2007, 7(3), 1028-1033.

[35] Husseini, G.A.; Pitt, W.G.; Christensen, D.A.; Dickinson, D., Degradation kinetics of stabilized pluronic micelles under the action of ultrasound. $J$. Control. Rel., 2009, 138, 45-48.

[36] Husseini, G.A.; Abdel-Jabbar, N.M.; Mjalli, F.S.; Pitt, W.G. Modeling and sensitivity analysis of acoustic release of doxorubicin from unstabilized pluronic P105 using an artificial neural network model. Technol. Cancer Res. Treatment, 2007, 6(1), 1-8.

[37] Stevenson-Abouelnasr, D.; Husseini, G.A.; Pitt, W.G. Further investigation of the mechanism of doxorubicin release from P105 micelles using kinetic models, Colloid Surf. B, 2007, 55, 59-66.

[38] Husseini, G.A.; Mjalli, F.S.; Pitt, W.G.; Abdel-Jabbar, N.M. Using artificial neural networks and model predictive control to optimize acoustically assisted doxorubicin release from polymeric micelles. Tech. Cancer Res. Treatment, 2009, 8(6), 479-488.

[39] Husseini, G.A.; Stevenson-Abouelnasr, D.; Pitt, W.G.; Assaleh, K.T.; Farahat, L.O.; Fahadi, J. Kinetics and thermodynamics of acoustic release of doxorubicin from non-stabilized polymeric micelles. Colloids Sur. A, 2010, 359, 18-24.

[40] Husseini, G.A., Diaz de la Rosa, M.A., AlAqqad, E.O., Al Mamary, S., Kadimanti, Y., Al Baik, A. Pitt, W.G. Kinetics of acoustic release of doxorubicin from stabilized and unstabilized micelles and the effect of temperature. J. Franklin I., 2011, 348(1), 125-133.

[41] Varshosaz, J.; Taymouri, S.; Hassanzadeh, F.; Haghjooy Javanmard, S.; Rostami, M., Folated Synperonic-Cholesteryl Hemisuccinate Polymeric Micelles for the Targeted Delivery of Docetaxel in Melanoma. BioMed Res. Int., 2015, 2015, 1-17.

[42] Kang, J.H.; Battogtokh, G.; Ko, Y.T. Folate-targeted liposome encapsulating chitosan/oligonucleotide polyplexes for tumor targeting. AAPS Pharm. Sci. Tech., 2014, 15(5), 1087-1092.

[43] Yoo, H.S.; Park, T.G., Folate-receptor-targeted delivery of doxorubicin nano-aggregates stabilized by doxorubicin-PEG- folate conjugate. J. Control. Release, 2004, 100 (2), 247-256.

[44] Zhao, H.; Yung, L. Y. L., Selectivity of folate conjugated polymer micelles against different tumor cells. Int. J. Pharm., 2008, 349 (1), 256-268.

[45] Amin, M.; Badiee, A.; Jaafari, M.R., Improvement of pharmacokinetic and antitumor activity of PEGylated liposomal doxorubicin by targeting with $N$ methylated cyclic RGD peptide in mice bearing C-26 colon carcinomas. Int. J. Pharm., 2013, 458(2), 324-333.

[46] Tsai, C.H.; Tsai, H.C.; Chang, C.H.; Chang, W.H.; Lo, C.L.; Ou, T.W.; Yen, T.C.; Hsiue, G.H. Graft and diblock copolymer multifunctional micelles for cancer chemotherapy and imaging. Biomaterials 2010, 31(8), 2293-2301.

[47] Kedar, U.; Phutane, P.; Shidhaye, S.; Kadam, V. Advances in polymeric micelles for drug delivery and tumor targeting. Nanomedicine, 2010, 6(6), 714729.

[48] Chen, C.; Ke, J.Y.; Zhou, X.E.; Yi, W.; Brunzelle, J.S.; Li, J.; Yong, E.L.; Xu, H.E.; Melcher, K. Structural basis for molecular recognition of folic acid by folate receptors. Nature, 2013, 500(7463), 486-486.

[49] Su, C.; Li, H.D.; Shi, Y.J.; Wang, G.; Liu, L. W.; Zhao, L.; Su, R.J., Carboxymethyl-beta-cyclodextrin conjugated nanoparticles facilitate therapy for folate receptor-positive tumor with the mediation of folic acid. Int. J. Pharm., 2014, 474 (1-2), 202-211.

[50] Wang, X.; Li, J.; Wang, Y.X.; Koenig, L.; Gjyrezi, A.;

Giannakakou, P.; Shin, E.H.; Tighiouart, M.; Chen, Z.; Nie, S. M.; Shin, D.M. A folate receptor-targeting nanoparticle minimizes drug resistance in a human cancer model. ACS Nano., 2011, 5(8), 61846194.

[51] Xue, Y.; Tang, X.; Huang, J.; Zhang, X.; Zhang, Y.; Yu, J.; Gui, S. Anti-tumor efficacy of polymer-platinum(II) complex micelles fabricated from folate conjugated PEG- graft- $\alpha, \beta$-poly [(N-amino acidyl)-aspartamide] and cis-dichlorodiammine platinum(II) in tumor-bearing mice. Colloid Surf. B: $B$, 2011, 85(2), 280-288.

[52] Hong, G.; Yuan, R.; Liang, B.; Shen, J.; Yang, X.; Shuai, X., Folate-functionalized polymeric micelle as hepatic carcinomatargeted, MRI-ultrasensitive delivery system of antitumor drugs.

Biomed. Microdevices, 2008, 10(5), 693-700. 
[53] Santra, S.; Kaittanis, C.; Santiesteban, O.J.; Perez, J.M. CellSpecific, activatable, and theranostic prodrug for dual-targeted cancer imaging and therapy. J. Am. Chem. Soc., 2011, 133 (41), 16680-16688.

[54] Husseini, G.A.; Kanan, S.; Al-Sayah, M. Investigating the fluorescence quenching of doxorubicin in folic acid solutions and its relation to ligandtargeted nanocarriers. J. Nanosci. Nanotechnol., 2015, 15, 1-5.

[55] Husseini, G.A. Folic Acid Quenches Doxorubicin Fluorescence" Letter to the Editor, Adv. Sci. Lett., 7, 726(2012).

[56] Liu, S.Q.; Wiradharma, N.; Gao, S.J.; Tong, Y.W.; Yang, Y.Y., Bio-functional micelles self-assembled from a folate-conjugated block copolymer for targeted intracellular delivery of anticancer drugs. Biomaterials, 2007, 28(7), 1423-1433.

[57] Yoo, H.S.; Park, T.G. Folate receptor targeted biodegradable polymeric doxorubicin micelles. J. Control. Release, 2004, 96(2), $273-283$. 